[3] D. V. Hutten and F. Hu, "Acoustic emission monitoring of tool wear in end milling using time-domain averaging," Trans. ASME, J. Manuf. Sci. Eng., vol. 121, pp. 8-12, 1999.

[4] X. Li and Z. Yuan, "Tool wear monitoring with wavelet packet transform-fuzzy clustering method," Wear, vol. 219, pp. 145-154, 1998.

[5] X. Li, "A brief review: acoustic emission for tool wear monitoring during turning," Int. J. Mach. Tools Manuf., vol. 42, no. 1, pp. 157-165, 2002.

[6] J. T. Roth and S. M. Pandit, "Monitorig end-mill wear and predicting tool failure using accelerometers," Trans. ASME, J. Manuf. Sci. Eng., vol. 121, no. 4, pp. 559-567, 1999.

[7] Y. Altintas, "Prediction of cutting forces and tool breakage in milling from feed drive current measurements," Trans. ASME, J. Eng. Ind., vol. 114, pp. 386-392, 1992.

[8] X. Li, D. Alexandar, and K. V. Patri, "Current sensor-based feed cutting force intelligent estimation and tool wear condition monitoring," IEEE Trans. Ind. Electron., vol. 47, pp. 697-702, July 2000.

[9] X. Li, "On-line detection of the breakage of small diameter drills using current signature wavelet transform," Int. J. Mach. Tools, Manuf., vol. 39, no. 1, pp. 157-164, 1999.

[10] X. Li, S. K. Tso, and J.Jun Wang, "Real-time tool condition monitoring using wavelet transforms and fuzzy techniques," IEEE Trans. Syst., Man, Cybern. C, vol. 31, pp. 352-357, Aug. 2000.

[11] Y.Yves Nievergelt, Wavelets Made Easy. Cambridge, MA: Birkhaauser, 1999.

[12] R. J. Jannarone, Concurrent learning and information processing. London, U.K.: Chapman \& Hall, 1997.

[13] X. Li, "Detection of tool flute breakage in end milling using feed-motor current signatures," IEEE/ASME Trans. Mechatron., vol. 6, pp. 491-498, Dec. 2001.

[14] R. Wichman, J. T. Astola, P. J. Heinonen, and Y. A. Neuvo, "FIR-median hybrid filters with excellent transient response in noisy conditions," IEEE Trans. Acoust., Speech, Signal Processing, vol. 38, pp. 2108-2117, Dec. 1990.

\section{Nonlinear Coupling Control Laws for an Underactuated Overhead Crane System}

\author{
Y. Fang, W. E. Dixon, D. M. Dawson, and E. Zergeroglu
}

\begin{abstract}
In this paper, we consider the regulation control problem for an underactuated overhead crane system. Motivated by recent passivitybased controllers for underactuated systems, we design several controllers that asymptotically regulate the planar gantry position and the payload angle. Specifically, utilizing LaSalle's invariant set theorem, we first illustrate how a simple proportional-derivative (PD) controller can be utilized to asymptotically regulate the overhead crane system. Motivated by the desire to achieve improved transient performance, we then present two nonlinear controllers that increase the coupling between the planar gantry position and the payload angle. Experimental results are provided to illustrate the improved performance of the nonlinear controllers over the simple PD controller.
\end{abstract}

Index Terms-Energy damping, Lyapunov methods, nonlinear control, overhead crane.

\section{INTRODUCTION}

Precise payload positioning by an overhead crane (especially when performed by an operator using only visual feedback to position the payload) is difficult due to the fact that the payload can exhibit a pendulum-like swinging motion. Motivated by the desire to achieve fast and precise payload positioning while mitigating performance and safety concerns associated with the swinging motion, several researchers have developed various controllers for overhead crane systems. For example, Yu et al. [29] utilized a time-scale separation approach to control an overhead crane system; however, an approximate linearized model of the crane was utilized to facilitate the construction of the error systems. In [27], Yashida et al. proposed a saturating control law based on a guaranteed cost control method for a linearized version of the crane system dynamics. Martindale et al. [18] utilized an approximate crane model to develop exact model knowledge and adaptive controllers while Butler et al. [2] exploited a modal decomposition technique to develop an adaptive controller. In [3], Chung and Hauser designed a nonlinear controller for regulating the swinging energy of the payload.

Several researchers have also examined the control problem for overhead crane systems with additional degree of freedom (DOF). Specifically, Moustafa and Ebeid [19] derived the nonlinear dynamic model for an overhead crane and then utilized a standard linear feedback controller based on a linearized state space model. In [20], Noakes and

Manuscript received September 19, 2001; revised July 17, 2002, and February 26, 2003. This work was supported in part the U.S. Department of Energy, in part by the Office of Biological and Environmental Research (OBER) Environmental Management Sciences Program (EMSP) under Project 82797, in part by the U.S. National Science Foundation under Grant DMI-9457967, in part by the Office of Naval Research under Grant N00014-99-1-0589, in part by the Department of Coomerce, and in part by the Army Research Office Automotive Center Grant.

Y. Fang is with the Sibley School of Mechanical and Aerospace Engineering, Cornell University, Ithaca, NY 14853-7501 USA (e-mail: yf47@ cornell.edu).

W. E. Dixon is with Oak Ridge National Laboratory, Oak Ridge, TN 37831 USA (e-mail: dixonwe@ornl.gov).

D. M. Dawson is with the Department of Electrical \& Computer Engineering, Clemson University, Clemson, SC 29634-0915 USA (e-mail: ddawson@ces.clemson.edu).

E. Zergeroglu is with the Department of Computer Engineering, Gebze Institute of Technology, 41400 Gebze-Kocaeli, Turkey (e-mail: ezerger@bilmuh.gyte.edu.tr).

Digital Object Identifier 10.1109/TMECH.2003.816822 
Jansen developed a generalized input shaping approach for the linearized crane dynamics that exploited a notch filtering technique to control the motion of the bridge/trolley of an overhead crane system. More recently, Lee [16] developed a nonlinear model for overhead cranes based on a new swing-angle definition. Based on this nonlinear model, Lee then developed an anti-swing control law for the decoupled linearized dynamics. In [22], Sakawa and Sano derived a nonlinear model for a crane system that was subsequently linearized to facilitate the development of a control scheme that first transferred the load to a position near the equilibrium point using an open-loop controller and then utilized a linear feedback controller to stabilize the payload about the equilibrium point.

One of the limiting factors associated with the above overhead crane control designs is that the system nonlinearities are often excluded from the closed-loop error system design and stability analysis. To overcome this drawback, several researchers have investigated control approaches that account for the nonlinear dynamics of overhead cranes and similar systems. For example in [24], Teel utilized saturation functions to develop an output feedback controller which achieves a robust, semi-global stability result for the ball-and-beam control problem. In [1], Burg et al. transformed the nonlinear crane dynamics into a structure that resembled the ball-and-beam problem and then adopted the research efforts of [24] to achieve asymptotic positioning from a large set of initial conditions. More recently, Fantoni et al. [8] and Lozano et al. [17] proposed passivity-based controllers for the inverted pendulum and the pendubot (i.e., an inverted pendulum-like robot with an unactuated second link) based on the paradigm of driving the underactuated system to a homoclinic orbit using an energy-based nonlinear controller and then switching to a linear controller to stabilize the system around its unstable equilibrium point. Using similar stability-analysis techniques, Collado et al. [4] proposed a proportional-derivative (PD) controller for an overhead crane problem with a 1-DOF gantry. In [15], Kiss et al. developed a PD controller for a vertical crane-winch system that only requires the measurement of the winch angle and its derivative rather than a cable angle measurement. In [12] and [21], passivity based interconnection and damping assignment control techniques are used to stabilize underactuated mechanical systems. Specifically, the desired inertia matrix is parameterized such that energy shaping can be exploited to stabilize underactuated mechanical systems such as the ball-and-beam problem and the inverted pendulum. In [28], Yoshida developed a nonlinear energy-based controller to damp out the pendulum oscillations despite amplitude constraints on the trolly position. Recently, in [5] and [6], Fang et al. developed several energy-based controllers for overhead crane systems in which additional nonlinear terms were injected into the controller to increase the coupling between the gantry position and the payload position to provide for improved transient response.

In this paper, a linear PD controller is explicitly proven to yield asymptotic regulation of an overhead crane system. By using similar analysis techniques as in [8] and [17], this paper provides the first explicit proof that a PD controller can be used to regulate the underactuated, nonlinear 2-DOF gantry and payload dynamics. Specifically, a PD feedback loop at the gantry creates an artificial spring/damper system which absorbs the payload energy. Motivated by the heuristic concept that improved coupling between the gantry and the payload dynamics will provide a mechanism for improved transient response, additional nonlinear feedback terms are incorporated with the PD controller and the resulting nonlinear controller is proven to yield gantry/payload regulation using similar analysis techniques. The design for one of the nonlinear controllers is motivated by the "energy-squared" approach inspired by [17]. A second nonlinear approach is also presented to illustrate that additional energy-based controllers can be developed that exploit the increased coupling between the gantry and payload for im-

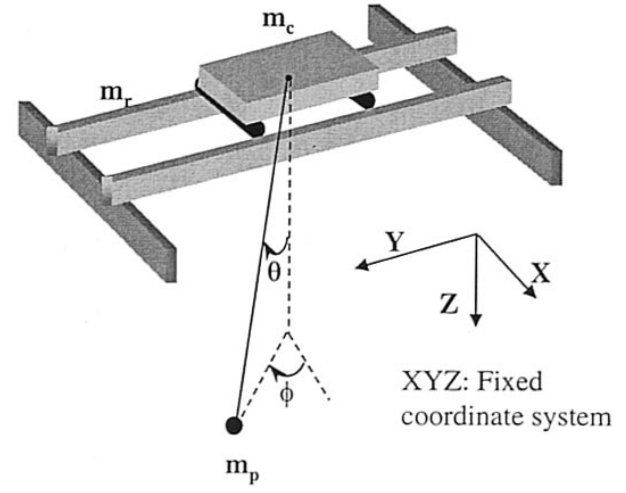

Fig. 1. Overhead crane system.

proved transient response. That is, another contribution of this research is to illustrate how nonlinear feedback terms can be incorporated in the control design to provide additional feedback for the unactuated payload angle through the natural coupling that exists between the gantry and the payload. To further examine the potential for improved transient performance, additional analysis techniques such as those presented in [11] could possibly be explored to bound the transient response. While additional analytical approaches such as [11] are beyond the scope of this paper, experimental results are provided, which illustrate that the increased coupling of the nonlinear controllers results in improved transient response over the linear PD control law.

\section{DYNAMIC MODEL}

The dynamic model of the underactuated overhead crane system (see Fig. 1) is assumed to have the following form [19]:

$$
M(q) \ddot{q}+V_{m}(q, \dot{q}) \dot{q}+G(q)=u .
$$

In (1), $M(q) \in \mathbb{R}^{4 \times 4}, V_{m}(q, \dot{q}) \in \mathbb{R}^{4 \times 4}$, and $G(q) \in \mathbb{R}^{4}$, represent the inertia, centripetal-Coriolis, and gravity terms (for details regarding the components of these matrices see [6], [7]), respectively, $q(t) \in \mathbb{R}^{4}$ is defined as follows:

$$
q=\left[\begin{array}{llll}
x & y & \theta & \phi
\end{array}\right]^{T}
$$

where $x(t) \in \mathbb{R}$ denotes the gantry position along the $X$-coordinate axis, $y(t) \in \mathbb{R}$ denotes the gantry position along the $Y$-coordinate axis, $\theta(t) \in \mathbb{R}$ denotes the payload angle with respect to the vertical, $\phi(t) \in$ $\mathbb{R}$ denotes the projection of the payload angle along the $X$-coordinate axis, and $u(t) \in \mathbb{R}^{4}$ is defined as

$$
u=\left[\begin{array}{llll}
F_{x} & F_{y} & 0 & 0
\end{array}\right]^{T}
$$

where $F_{x}(t)$ and $F_{y}(t) \in \mathbb{R}$ represent the control-force inputs acting on the cart and rail, respectively. Based on the structure of $M(q)$ and $V_{m}(q, \dot{q})$, it is straightforward to show that the following skew-symmetric relationship is satisfied:

$$
\xi^{T}\left(\frac{1}{2} \dot{M}(q)-V_{m}(q, \dot{q})\right) \xi=0 \quad \forall \xi \in \mathbb{R}^{4}
$$

where $\dot{M}(q)$ represents the time derivative of $M(q)$, and that the inertia matrix $M(q)$ can be upper and lower bounded by the following inequalities:

$$
k_{1}\|\xi\|^{2} \leq \xi^{T} M(q) \xi \leq k_{2}\|\xi\|^{2} \quad \forall \xi \in \mathbb{R}^{4}
$$

where $k_{1}$ and $k_{2} \in \mathbb{R}$ are positive bounding constants. In a similar manner as in [1] and [8], we assume that the dynamic model given in (1) has the following characteristics. 
Assumption 1: The payload and the gantry are connected by a massless, rigid link.

Assumption 2: The angular position and velocity of the payload and the planar position and velocity of the gantry are measurable.

Assumption 3: The gantry mass and the length of the connecting rod are known.

Assumption 4: The connection between the payload link and the gantry is frictionless and does not rotate about the connecting link (i.e., the payload does not rotate about the link axis).

Assumption 5: The angular position of the payload mass is restricted according to the following inequality:

$$
-\pi<\theta(t)<\pi
$$

where $\theta(t)$ is measured from the vertical position (see Fig. 1).

Remark 1: Note that the model given by (1) could be modified to include other dynamic effects associated with the gantry dynamics (e.g. gantry friction, viscous damping coefficients, mass moment of inertia of the gantry and rail motors, etc.); however, these additional dynamic effects were not included in the model since these effects can be directly cancelled by the controller.

\section{OPEN-LOOP SYSTEM DEVELOPMENT}

To express (1) in a form that facilitates the subsequent control development and stability analysis, we premultiply both sides of (1) by $M^{-1}(q)$ to obtain the following expression:

$$
\ddot{q}=M^{-1}\left(u-V_{m} \dot{q}-G\right)
$$

where $M^{-1}(q) \in \mathbb{R}^{4 \times 4}$ is guaranteed to exist due to the fact that the determinant of $M(q)$, denoted by $\operatorname{det}(M)$, is a positive function (see [6] and [7] for explicit details). After performing some algebraic manipulation, the first two rows of (7) can be expressed as follows:

$$
\begin{aligned}
& \ddot{x}=\frac{1}{\operatorname{det}(M)}\left(p_{11} F_{x}+p_{12} F_{y}+w_{1}\right) \\
& \ddot{y}=\frac{1}{\operatorname{det}(M)}\left(p_{12} F_{x}+p_{22} F_{y}+w_{2}\right)
\end{aligned}
$$

where the measurable terms $p_{11}(q), p_{12}(q), p_{22}(q), w_{1}(q, \dot{q})$, $w_{2}(q, \dot{q}) \in \mathbb{R}$ are defined as follows:

$$
\begin{aligned}
p_{11}= & m_{p}^{2} L^{2} I\left(\sin ^{2} \phi+2 \sin ^{2} \theta \cos ^{2} \phi\right)+m_{p} I^{2} \\
& +m_{c} m_{p} L^{4} \sin ^{2} \theta+m_{c} m_{p} L^{2} I\left(1+\sin ^{2} \theta\right) \\
& +m_{c} I^{2}+m_{p}^{3} L^{4} \cos ^{2} \phi \sin ^{4} \theta \\
p_{12}= & -m_{p}^{3} L^{4} \sin \phi \cos \phi \sin ^{4} \theta \\
& -m_{p}^{2} L^{2} I \sin \phi \cos \phi\left(\sin ^{2} \theta-\cos ^{2} \theta\right) \\
p_{22}= & m_{p}^{3} L^{4} \sin ^{4} \theta \sin ^{2} \phi \\
& +m_{p}^{2} L^{2} I\left[1+\left(\sin ^{2} \theta-\cos ^{2} \theta\right) \sin ^{2} \phi\right] \\
& +\left(m_{p}+m_{r}+m_{c}\right) I^{2}+\left(m_{r}+m_{c}\right) m_{p}^{2} L^{4} \sin ^{2} \theta \\
& +\left(m_{r}+m_{c}\right) m_{p} L^{2} I\left(1+\sin ^{2} \theta\right) \\
w_{1}= & m_{p} L \sin \theta \sin \phi \\
& \cdot\left[\left(m_{p}+m_{c}\right) I+m_{p} m_{c} L^{2} \sin ^{2} \theta\right] \\
& \cdot\left[\dot{\phi}^{2}\left(m_{p} L^{2} \sin { }^{2} \theta+I\right)+\dot{\theta}^{2}\left(m_{p} L^{2}+I\right)\right] \\
& -2 I m_{p} L \dot{\theta} \dot{\phi} \cos \theta \cos \phi \\
& \cdot\left[\left(m_{p}+m_{c}\right) I+m_{p} L^{2}\left(m_{c}+m_{p} \sin ^{2} \theta\right)\right] \\
& +m_{p}^{2} g L^{2} \sin \theta \cos \theta \sin \phi \\
& \cdot\left[\left(m_{p}+m_{c}\right) I+m_{p} m_{c} L^{2} \sin ^{2} \theta\right]
\end{aligned}
$$

$$
\begin{aligned}
w_{2}= & m_{p} L \sin \theta \cos \phi \\
& \cdot\left[\left(m_{p}+m_{r}+m_{c}\right) I+\left(m_{r}+m_{c}\right) m_{p} L^{2} \sin ^{2} \theta\right] \\
& \cdot\left[d^{2}\left(m_{p} L^{2} \sin ^{2} \theta+I\right)+p^{2}\left(m_{p} L^{2}+I\right)\right] \\
& +2 m_{p} L I \dot{\theta} \dot{\phi} \cos \theta \sin \phi \\
& \cdot\left[\left(m_{p}+m_{r}+m_{c}\right)\left(m_{p} L^{2}+I\right)-m_{p}^{2} L^{2} \cos ^{2} \theta\right] \\
& +m_{p} g L \sin \theta\left[\left(m_{r}+m_{c}\right) m_{p}^{2} L^{3} \sin ^{2} \theta \cos \theta \cos \phi\right. \\
& \left.+\left(m_{r}+m_{c}+m_{p}\right) m_{p} L I \cos \theta \cos \phi\right]
\end{aligned}
$$

where $m_{p}, m_{r}$, and $m_{c} \in \mathbb{R}$ represent the payload mass, rail mass, and cart mass, respectively, $I \in \mathbb{R}$ denotes the moment of inertia of the payload, $L \in \mathbb{R}$ represents the length of the crane rod, and $g \in$ $\mathbb{R}$ represents the gravity constant. To write the open-loop dynamics given in (8) and (9) in a more compact form for the subsequent control development and stability analysis, we define $r(t) \in \mathbb{R}^{2}$ as follows:

$$
r=\left[\begin{array}{ll}
x & y
\end{array}\right]^{T} .
$$

After taking the second time derivative of $r(t)$, we can rewrite the open-loop dynamics given in (8) and (9) as follows:

$$
\ddot{r}=\left[\begin{array}{l}
\ddot{x} \\
\ddot{y}
\end{array}\right]=\frac{1}{\operatorname{det}(M)}(P F+W)
$$

where $P(q) \in \mathbb{R}^{2 \times 2}$ and $W(q, \dot{q}) \in \mathbb{R}^{2}$ are defined as follows:

$$
P=\left[\begin{array}{ll}
p_{11} & p_{12} \\
p_{12} & p_{22}
\end{array}\right] \quad W=\left[\begin{array}{l}
w_{1} \\
w_{2}
\end{array}\right]
$$

and $F(t) \in \mathbb{R}^{2}$ is defined as

$$
F=\left[\begin{array}{ll}
F_{x} & F_{y}
\end{array}\right]^{T} .
$$

Based on (17) and the fact that $P(q)$ is a leading minor of the positive-definite matrix $M^{-1}$, we can conclude that $P(q)$ is positive definite, symmetric, and invertible, where the inverse of $P(q)$, denoted by $P^{-1}(q)$, is also positive definite and symmetric [13].

To facilitate the subsequent Lyapunov-based control design, we utilize the energy of the overhead crane system, denoted by $E(q, \dot{q}) \in \mathbb{R}$, defined as follows:

$$
E(q, \dot{q})=\frac{1}{2} \dot{q}^{T} M(q) \dot{q}+m_{p} g L(1-\cos (\theta)) \geq 0 .
$$

After taking the time derivative of (19), substituting (1) for $M(q) \ddot{q}(t)$, utilizing (4), and canceling common terms, the following expression can be obtained:

$$
\dot{E}=\dot{r}^{T} F \text {. }
$$

\section{CONTROL DESIGN AND ANALYSIS}

The control objective in this paper is to regulate the planar gantry position of the overhead crane to a constant desired position, denoted by $r_{d} \in \mathbb{R}^{2}$, which is explicitly defined as

$$
r_{d}=\left[\begin{array}{ll}
x_{d} & y_{d}
\end{array}\right]^{T}
$$

while simultaneously regulating the payload angle $\theta(t)$ to zero. To quantify the control objective of regulating the overhead crane to a constant desired position, we define a gantry position error signal $e(t) \epsilon$ $\mathbb{R}^{2}$ as follows:

$$
e(t)=r-r_{d}
$$


In the subsequent control development, a PD control law and two nonlinear controllers are designed and proven to achieve the above control objective through a Lyapunov-based stability analysis.

Remark 2: As in [19], the crane dynamic model given in (1)-(3) exploits a projection of the payload angle along the $X$-Coordinate axis, denoted by $\phi(t)$. By injecting this artificial state, the dynamic model can be written in a manner that facilitates the development of controllers that achieve the control objective; unfortunately, the overall stability analysis is complicated by the fact that another unactuated state is injected into the system. From a physical standpoint, if the payload angle, denoted by $\theta(t)$, is regulated to zero, then from Fig. 1, the payload is regulated to the desired location, and hence, the control objective is not defined in terms of regulating $\phi(t)$. Despite this theoretical issue, simulation results and the experimental results provided in Section V illustrate that the subsequently developed controllers provide superior transient performance with respect to a simple PD controller.

\section{A. PD Control Law}

Based on the subsequent stability analysis, we design the following PD control law:

$$
F=-\left(k_{d} \dot{r}+k_{p} e\right)
$$

where $k_{d}$ and $k_{p} \in \mathbb{R}$ are positive constant control gains.

Theorem 1: The controller given in (23) ensures asymptotic regulation of the overheadcrane system in the sense that

$$
\lim _{t \rightarrow \infty}(x(t) \quad y(t) \quad \theta(t))=\left(\begin{array}{lll}
x_{d} & y_{d} & 0
\end{array}\right)
$$

where $x_{d}$ and $y_{d}$ were defined in (21).

Proof: To prove (24), we define a nonnegative function $V_{1}(t) \in$ $\mathbb{R}$ as follows:

$$
V_{1}=E+\frac{1}{2} k_{p} e^{T} e
$$

After taking the time derivative of (25) and then substituting (20), the time derivative of (22), and (23) into the resulting expression and then cancelling common terms, we obtain the following:

$$
\dot{V}_{1}=-k_{d} \dot{r}^{T} \dot{r}
$$

Based on the expressions given in (5), (19), (22), (25), and (26), it is clear $r(t), e(t)$, and $\dot{q}(t) \in \mathcal{L}_{\infty}$. Based on the fact that $r(t), e(t)$, $\dot{q}(t) \in \mathcal{L}_{\infty}$, it can be determined from (2) and (15) that $x(t), \dot{x}(t)$, $y(t), \dot{y}(t), \dot{r}(t), \dot{\theta}(t)$, and $\dot{\phi}(t) \in \mathcal{L}_{\infty}$. Given that $e(t)$ and $\dot{r}(t) \in \mathcal{L}_{\infty}$, it is clear from (23) that $F(t) \in \mathcal{L}_{\infty}$. Finally, from (3) and (18), we can prove that $F_{x}(t), F_{y}(t), u(t) \in \mathcal{L}_{\infty}$.

Based on the fact that all of the closed-loop signals remain bounded, LaSalle's Invariance Theorem can now be utilized to prove (24). To this end, we define $\Gamma$ as the set of all points where

$$
\dot{V}_{1}=0 \text {. }
$$

In the set $\Gamma$, it is clear from (26) and (27) that

$$
\dot{r}(t)=0 \quad \ddot{r}(t)=0
$$

and hence, we can conclude from (15), (25), (27), and (28) that $x(t)$, $y(t)$, and $V_{1}(t)$ are constant. Furthermore, from (20), (22), and (28), it is clear that

$$
\dot{E}(q, \dot{q})=\dot{e}(t)=0 .
$$

Based on (29), it is clear that $E(q, \dot{q})$ and $e(t)$ are constant, and hence, from (23) and (28), it is clear that $F(t)$ is constant. To complete the proof, the stability of the system must be analyzed for the case when $\dot{\theta}(t)=0 \forall t$ and the case when $\dot{\theta}\left(t_{0}\right) \neq 0$ for a specific time $t_{0}$. Specifically, the result given in (24) can be proven under the proposition that $\dot{\theta}(t)=0 \forall t$ (see [7] for the detailed analysis). Development is also provided in [7] to prove that the proposition $\dot{\theta}\left(t_{0}\right) \neq 0$ for a specific time $t_{0}$ leads to a contradiction, and hence, is invalid.

Remark 4: In the stability analysis for Theorem 1, we have shown that the control objective is met and that all signals in the dynamics and the controller remain bounded for all time except for the signal $\phi(t)$ (Note that by assumption, the payload angle, denoted by $\theta(t)$, is assumed to be bounded). We note that the boundedness of $\phi(t)$ is insignificant from a theoretical point of view since $\phi(t)$ only appears in the dynamics and control as arguments of trigonometric functions.

Remark 5: Heuristically, the only way for the energy from the payload motion to be dissipated is through the coupling between the payload dynamics and the gantry dynamics. That is, a PD feedback loop at the gantry creates an artificial spring/damper system which absorbs the payload energy through the natural gantry/payload coupling; however, from our experience with many control experiments on overhead crane testbeds, we believe that a PD feedback loop at the gantry will always provide poor performance because the gantry friction is not compensated for perfectly (and it never will be). That is, the uncompensated gantry friction effects tend to retard the natural coupling between the gantry/payload dynamics, and hence, prevent payload energy from being dissipated by the PD feedback loop at the gantry. In the following sections, we present controllers that tend to improve the performance of the PD feedback loop at the gantry due to the incorporation of additional nonlinear terms in the control law that depend on the payload dynamics. Although the subsequent controllers yield the same stability result as the PD controller, the increase in gantry/payload coupling due to the additional nonlinear terms results in improved performance when compared to the simple gantry PD controller. That is, the subsequent controllers exploit additional energy-based nonlinear terms which provide increased payload swing feedback through the coupling between the gantry and the payload. This concept has also been explored in [9] and [10]. The improved performance of the subsequent controllers has been demonstrated by simulation results and by the experimental results presented in Section V.

\section{B. Nonlinear Coupling Control Laws}

Based on previous work presented in [17] for an inverted pendulum, we design the following: $E^{2}$ coupling control law:

$$
F=[\Omega]^{-1}\left(-k_{d} \dot{r}-k_{p} e-\frac{k_{v}}{\operatorname{det}(M)} W\right)
$$

where $\Omega(t) \in \mathbb{R}^{2 \times 2}$ is an auxiliary positive-definite, invertible matrix ${ }^{2}$ defined as follows:

$$
\Omega=k_{E} E I_{2}+\frac{k_{v}}{\operatorname{det}(M)} P
$$

$k_{E}, k_{p}, k_{d}, k_{v} \in \mathbb{R}$ are positive constant control gains, $I_{2}$ denotes the standard $2 \times 2$ identity matrix, and $P(q)$, and $W(q, \dot{q})$ were defined in (17). Using the same principles as in the the proof for Theorem 1, the $E^{2}$ coupling control law given in (30) can be proven to asymptotically

${ }^{1}$ The control strategy is called an $E^{2}$ coupling control law because its structure is motivated by a squared energy term and an additional squared gantry velocity term in the Lyapunov function.

${ }^{2}$ Since $\mathrm{P}$ and $I_{2}$ are positive definite matrices, and $k_{E}, k_{v}, E(q, \dot{q})$, and $\operatorname{det}(M(q))$ are positive scalars, it can be proven that $\Omega(t)$ is positive definite and invertible. 

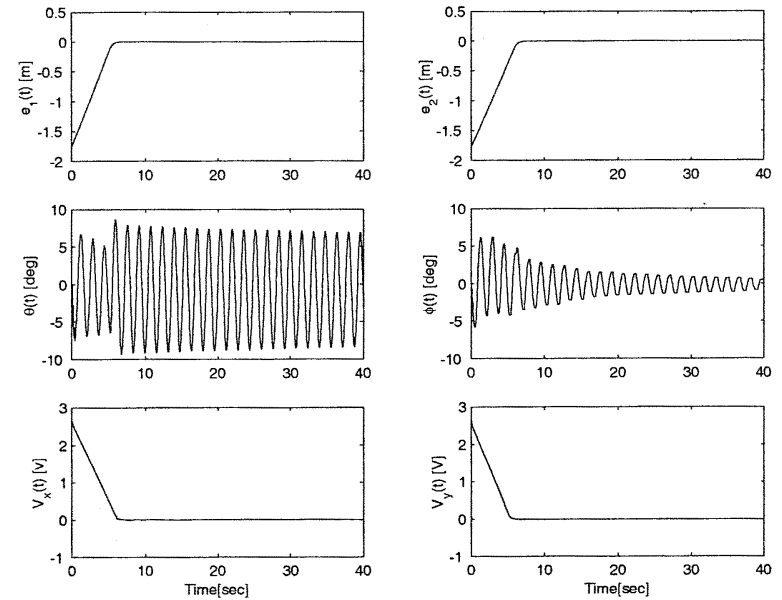

Fig. 2. Results for the PD controller

regulate the crane system. For explicit details that prove the controller given in (30) yields asymptotic regulation of the crane system see [7]. To illustrate how additional controllers can also be derived, we design the following nonlinear coupling control law: ${ }^{3}$

$$
F=\frac{-k_{d} \dot{r}-k_{p} e-k_{v} P^{-1} W-\frac{1}{2} k_{v}\left(\frac{d}{d t}\left(\operatorname{det}(M) P^{-1}\right)\right) \dot{r}}{k_{E}+k_{v}}
$$

where $k_{E}, k_{p}, k_{d}$, and $k_{v} \in \mathbb{R}$ are positive constant control gains, and $P(q)$, and $W(q, \dot{q})$ were defined in (17). The gantry kinetic energy coupling control law given in (32) can also be proven to yield the same stability result as described by Theorem 1. For explicit details that prove the controller given in (32) yields asymptotic regulation of the crane system see [7].

\section{EXPERIMENT RESULTS}

The controllers given in (23), (30), and (32) were implemented on the InTeCo overhead crane testbed [14]. The physical parameters of the overhead crane testbed were determined as follows:

$$
\begin{aligned}
m_{p} & =0.73 \mathrm{~kg} \\
m_{c} & =1.06 \mathrm{~kg} \\
m_{r} & =6.4 \mathrm{~kg} \\
I & =0.005 \mathrm{~kg} \cdot \mathrm{m}^{2} \\
L & =0.7 \mathrm{~m} .
\end{aligned}
$$

Each of the controllers was implemented at a sampling frequency of $1 \mathrm{kHz}$ and the desired gantry position was selected as follows:

$$
\left[\begin{array}{ll}
x_{d} & y_{d}
\end{array}\right]^{T}=\left[\begin{array}{ll}
1.75 & 1.75
\end{array}\right]^{T} \mathrm{~m} .
$$

For each of the experiments, the initial conditions were set to zero and the control gains were tuned until the best performance was achieved. The resulting gantry position error, payload angle, and the input force are shown in Fig. 2 for the PD control law, Fig. 3 for the $E^{2}$ coupling control law, and Fig. 4 for the gantry kinetic energy coupling

\footnotetext{
${ }^{3}$ The control strategy is called a gantry kinetic energy coupling control law because its structure is derived from an additional gantry kinetic energy-like term in the Lyapunov function.
}
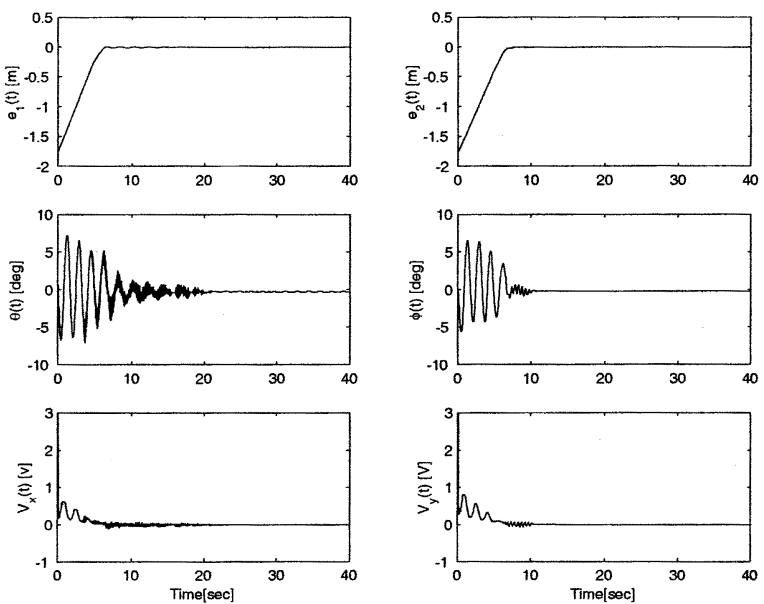

Fig. 3. Results for the $E^{2}$ coupling control law.
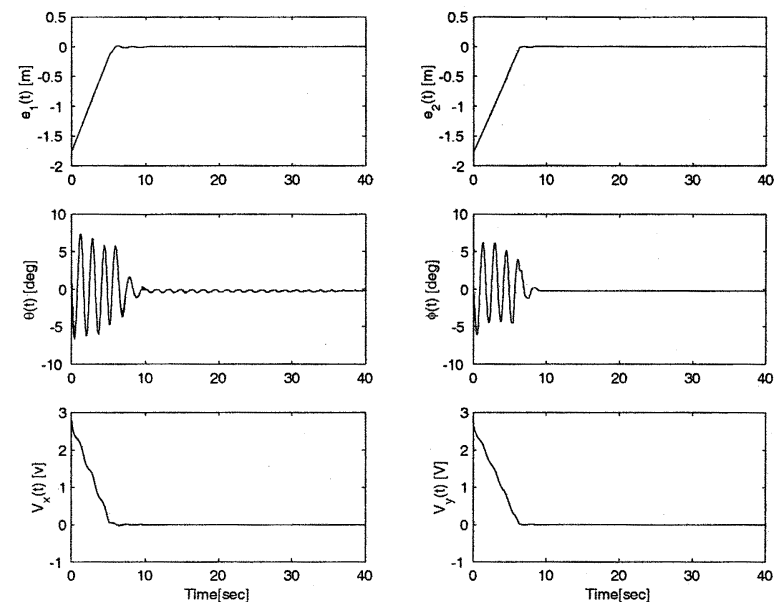

Fig. 4. Results for the gantry kinetic energy coupling contol law.

control law. Based on the results illustrated in Figs. 2-4 (more quantitative results are provided in [7]), it is clear that the coupling control laws exhibit superior settling time when compared to the simple PD controller. One reason for this superior performance is that the only payload/gantry coupling that exists for the PD controller is the natural coupling. When the gantry approaches the desired setpoint, friction damps the gantry to the extent that no overshoot can be observed; hence, the gantry stops and the payload swings freely. In contrast, the payload/gantry coupling for the coupling control laws is enhanced, resulting in gantry overshoot that damps out the payload swing.

\section{CONCLUSION}

In this paper, we presented three controllers for an overhead crane system. By utilizing a Lyapunov-based stability analysis along with LaSalle's Invariance Theorem, we proved asymptotic regulation of the gantry and payload position for a PD controller and two nonlinear controllers. Experimental results were utilized to demonstrate that the increased coupling between the gantry and payload that results from the additional nonlinear feedback terms in the nonlinear coupling control laws, resulted in improved transient response. Future efforts will focus on examining other types of overhead crane systems. For example, future efforts will target incorporating additional DOF such as the length of the pendulum link (i.e., height of the payload). Furthermore, future efforts will be directed at different modeling techniques that do not incorporate additional auxiliary states in the system. 


\section{REFERENCES}

[1] T. Burg, D. Dawson, C. Rahn, and W. Rhodes, "Nonlinear control of an overhead crane via the saturating control approach of teel," in Proc. IEEE Int. Conf. Robotics and Automation, 1996, pp. 3155-3160.

[2] H. Butler, G. Honderd, and J. Van Amerongen, "Model reference adaptive control of a gantry crane scale model," IEEE Contr. Syst. Mag., vol. 11, pp. 57-62, Jan. 1991.

[3] C. Chung and J. Hauser, "Nonlinear control of a swinging pendulum," Automatica, vol. 31, no. 6, pp. 851-862, 1995.

[4] J. Collado, R. Lozano, and I. Fantoni, "Control of convey-crane based on passivity," in Proc. American Control Conf., 2000, pp. 1260-1264.

[5] Y. Fang, E. Zergeroglu, W. E. Dixon, and D. M. Dawson, "Nonlinear coupling control laws for an overhead crane system," in Proc. IEEE Conf. Control Applications, Sept. 2001, pp. 639-644.

[6] Y. Fang, W. E. Dixon, E. Zergeroglu, and D. M. Dawson, "Nonlinear coupling control laws for a 3-DOF overhead crane system," in Proc. IEEE Conf.Decision and Control, Dec. 2001, pp. 3766-3771.

[7] Y. Fang, "Lyapunov-Based Control for Mechanical and Vision-Based Systems," Ph.D. dissertation, Clemson Univ., Clemson, SC, 2002.

[8] I. Fantoni, R. Lozano, and M. W. Spong, "Energy based control of the pendubot," IEEE Trans. Automat. Contr., vol. 45, pp. 725-729, Apr. 2000.

[9] S. S. Ge, T. H. Lee, and G. Zhu, "Asymptotically stable end-point regrlation of a flexible SCARA/Cartesian robot," IEEE/ASME Trans. Mechatron., vol. 3, pp. 138-144, June 1998.

[10] S. S. Ge, T. H. Lee, and Z. P. Wang, "Model-free regulation of multi-link flexible smart materials robots," IEEE/ASME Trans. Mechatron., vol. 6, pp. 346-351, Sept. 2001.

[11] S. S. Ge, C. C. Hang, and T. Zhang, "A direct adaptive controller for dynamics systems with a class of nonlinear parameterizations," Automatica, vol. 35, no. 4, pp. 741-747, 1999.

[12] F. Gomez-Estern, R. Ortega, F. R. Rubio, and J. Acacil, "Stabilization of a class of underactuated mechanical systems via total energy shaping," in Proc. Conf. Decision and Control, 2001, pp. 1137-1143.

[13] R. Horn and C. Johnson, Matrix Analysis. Cambridge, U.K.: Cambridge Univ. Press, 1985.

[14] 3DCrane: Installation and Commissioning Version 1.2, InTeCo, Krakow, Poland, 2000

[15] B. Kiss, J. Levine, and P. Mullhaupt, "A simple output feedback PD controller for nonlinear cranes," in Proc. Conf. Decision and Control, 2000 , pp. $5097-5101$.
[16] H. Lee, "Modeling and control of a three-dimensional overhead cranes," ASME Trans. Dyn. Syst. Meas. Control, vol. 120, pp. 471-476, 1998.

[17] R. Lozano, I. Fantoni, and D. J. Block, "Stabilization of the inverted pendulum around its homoclinic orbit," Syst. Control Lett., vol. 40, no. 3, pp. 197-204, 2000.

[18] S. C. Martindale, D. M. Dawson, J. Zhu, and C. Rahn, "Approximate nonlinear control for a two degree of freedom overhead crane: theory and experimentation," in Proc. American Control Conf., 1995, pp. 301-305.

[19] K. A. F. Moustafa and A. M. Ebeid, "Nonlinear modeling and control of overhead crane load sway," ASME Trans. Dyn. Syst. Meas. Control, vol. 110 , pp. 266-271, 1988.

[20] M. W. Noakes and J. F. Jansen, "Generalized inputs for damped-vibration control of suspended payloads," Robot. Autonomous Syst., vol. 10, pp. 199-205, 1992

[21] R. Ortega, M. W. Spong, and F. Gomez-Estern, "Stabilization of a class of underactuated mechanical systems via interconnection and damping assignment," IEEE Trans. Automat. Contr., vol. 47, pp. 1218-1233, Aug. 2002.

[22] Y. Sakawa and H. Sano, "Nonlinear model and linear robust control of overhead traveling cranes," Nonlinear Anal., vol. 30, no. 4, pp. 2197-2207, 1997.

[23] J. J. E. Slotine and W. Li, Applied Nonlinear Control. Englewood Cliffs, NJ: Prentice-Hall, 1991

[24] A. R. Teel, "Semi-global stabilization of the 'ball and beam' using 'output' feedback," in Proc. American Control Conf., 1993, pp. 2577-2581.

[25] Q. Wei, W. P. Dayawansa, and W. S. Levine, "Nonlinear controller for an inverted pendulum having restricted travel," Automatica, vol. 31, no. 6, pp. 841-850, 1995.

[26] Z. Yao, N. P. Costescu, S. P. Nagarkatti, and D. M. Dawson, "Real-time linux target: a MATLAB-based graphical control environment," in Proc. IEEE Conf. Control Applications, Anchorage, AK, 2000, pp. 173-178.

[27] K. Yoshida and H. Kawabe, "A design of saturating control with a guaranteed cost and its application to the crane control system," IEEE Trans. Automat. Contr., vol. 37, pp. 121-127, Aug. 1992.

[28] K. Yoshida, "Nonlinear controller design for a crane system with state constraints," in Proc. American Control Conf., 1998, pp. 1277-1283.

[29] J. Yu, F. L. Lewis, and T. Huang, "Nonlinear feedback control of a gantry crane," in Proc. American Control Conf., Seattle, WA, 1995, pp. $4310-4315$ 